\title{
Quantum Hydrodynamics, Quantum Benjamin-Ono Equation, and Calogero Model
}

\author{
Alexander G. Abanov ${ }^{1}$ and Paul B. Wiegmann ${ }^{2}$ \\ ${ }^{1}$ Department of Physics and Astronomy, Stony Brook University, Stony Brook, NY 11794 \\ ${ }^{2}$ James Franck Institute of the University of Chicago, 5640 S.Ellis Avenue, Chicago, IL 60637,
}

(Dated: September 10, 2018)

\begin{abstract}
Collective field theory for Calogero model represents particles with fractional statistics in terms of hydrodynamic modes - density and velocity fields. We show that the quantum hydrodynamics of this model can be written as a single evolution equation on a real holomorphic Bose field - quantum integrable Benjamin-Ono equation. It renders tools of integrable systems to studies of nonlinear dynamics of $1 \mathrm{D}$ quantum liquids.
\end{abstract}

1. Introduction. Calogero-Sutherland model occupies a special place in 1D quantum physics. A singular interaction (an inverse square potential) represents a fractional statistics of particles (for a review see e.g., 1]). The eigenfunctions of the model are neither symmetric nor antisymmetric. They have a form $(\Delta(x))^{\lambda} J(x)$, where $\Delta$ is a Vandermonde determinant and $J$ are symmetric polynomials (Jack polynomials). Although the problem does not possess a conformal invariance, it is intrinsically related to a boundary conformal field theory (CFT) 2] with a central charge $c=1-24 \alpha_{0}^{2}$, where $2 \alpha_{0}=\sqrt{\lambda}-1 / \sqrt{\lambda}$. Here we focus on a rational degeneration of the model - the Calogero model (CM)

$$
\mathcal{H}=\frac{1}{2} \sum_{j=1}^{N} p_{j}^{2}+\frac{1}{2} \sum_{j, k=1 ; j \neq k}^{N} \frac{\lambda(\lambda-1)}{\left(x_{j}-x_{k}\right)^{2}} .
$$

One might also add a harmonic potential $\frac{1}{2} \omega^{2} \sum_{i} x_{i}^{2}$ to prevent particles from running to infinity. Most of the formulas below are simple to generalize to the chiral sector of the trigonometric version of the model.

A productive approach to Calogero model in the limit of an infinite number of particles is a collective field theory developed by Sakita and Jevicki in Refs. 3, 4] and extended to $\mathrm{CM}$ in Refs. 2, 5]. In this approach an entire quantum theory with all the richness of an operator content, is represented as a quantum dissipationless hydrodynamics [6], i.e., solely in terms of the density $\rho(x)=\sum_{i} \delta\left(x-x_{i}\right)$ and the velocity operators

$$
[\rho(x), v(y)]=-i \delta^{\prime}(x-y) .
$$

Hydrodynamic approach is especially useful if one is interested in the evolution of smooth wave packets, larger than an inter-particle distance. Original particles apear in this approach as solitons of nonlinear fields.

In this letter we show that the quantum hydrodynamics (aka bosonization, or collective field theory) of Calogero model is equivalent to a quantum version of an integrable Benjamin-Ono equation on the double (QBO). The latter is a minor generalization of the conventional Benjamin-Ono equation (BO) arising in the hydrodynamics of stratified fluids [7]. A connection of the traditional BO to a complexified version of a classical Calogero model is known [8]. A pair of coupled classical BO-type equations has been obtained by A. Jevicki for a long-wave description of free fermions $(\lambda=1)[4]$.

2. Quantum hydrodynamics and non-linear bosonization. Collective or hydrodynamic approach is a nonlinear version of the bosonization procedure [9] - a popular method in low dimensional physics. In this approach, a fermionic spectrum is not linearized at the Fermi level. Therefore, an asymmetry of the particle-hole spectrum a dispersion of hydrodynamics modes - is not neglected. The price is a nonlinear and dispersive character of the hydrodynamics. The gain is a realm of nonlinear phenomena missed in a linear approximation. Before proceeding to the Quantum BO equation on the double (QBO), we start with the quantum hydrodynamics of free fermions.

Quantum hydrodynamics of free fermions with quadratic dispersion $(\lambda=1)$ can be obtained using conventional "bosonization". Writing a fermion $\psi(x)=$ $e^{i \varphi_{R}(x)}+e^{i \varphi_{L}(x)}$ as a superposition of left and right chiral fields $\left[\partial_{x} \varphi_{L, R}(x), \varphi_{L, R}(0)\right]=\mp i \delta(x)$ one obtains

$$
2 \pi(n+1) \psi^{\dagger}\left(-i \partial_{x}\right)^{n} \psi \sim:\left(\partial_{x} \varphi_{R}\right)^{n+1}-\left(\partial_{x} \varphi_{L}\right)^{n+1}:
$$

modulo full derivatives. Here and below we assume normal ordering. The first three moments are density $\rho=$ $\frac{1}{2 \pi} \partial_{x}\left(\varphi_{R}-\varphi_{L}\right)$, current $j=\rho v=\frac{1}{4 \pi}\left(\left(\partial_{x} \varphi_{R}\right)^{2}-\left(\partial_{x} \varphi_{L}\right)^{2}\right)$, and Hamiltonian density

$$
H=\frac{1}{12 \pi}\left(\left(\partial_{x} \varphi_{R}\right)^{3}-\left(\partial_{x} \varphi_{L}\right)^{3}\right)=\frac{1}{2} \rho v^{2}+\frac{\pi^{2}}{6} \rho^{3} .
$$

The operator $v=\frac{1}{2} \partial_{x}\left(\varphi_{R}+\varphi_{L}\right)$ is a velocity operator. The meaning of the terms in the energy (3) is clear. The "kinetic energy" reflects the Galilean invariance of (11), the "potential energy" reflects the fermionic statistics: $\left(\pi^{2} / 6\right) \rho^{3}$ is an energy density of a Fermi gas. This Hamiltonian takes into account all long-wave correlation properties of fermions, missing the physics at $2 k_{F}$ (e.g., Friedel oscillations), where the chiral sectors interact. Linearization at the equilibrium density $\rho_{0}=k_{F} / \pi$ leads to a familiar linear bosonization $H \approx$ $\frac{\rho_{0}}{4}\left(\left(\partial_{x} \varphi_{R}\right)^{2}+\left(\partial_{x} \varphi_{L}\right)^{2}\right)=\frac{\rho_{0}}{2}\left(v^{2}+\pi^{2}\left(\rho-\rho_{0}\right)^{2}\right)$. This approximation misses an interaction and dispersion of hydrodynamics modes, essential in many applications. 
The Hamiltonian (3) generates operator equations of quantum hydrodynamics 6

continuity equation :

$$
\begin{gathered}
\dot{\rho}+\partial_{x}(\rho v)=0 \\
\dot{v}+\partial_{x}\left(\frac{1}{2} v^{2}+w\right)=0
\end{gathered}
$$

where $\quad w=\delta(\rho \epsilon) / \delta \rho$ is the quantum enthalpy and $\epsilon=$ $\left(\pi^{2} / 6\right) \rho^{2}$ is the energy of a filled Fermi sea per particle.

3. Holomorphic Bose field. Both continuity equation (4) and Euler equation (5) for free fermions can be compactly written in terms of a single holomorphic field $u(z, t)$. It is a real field obeying the Schwarz reflection symmetry $u(z)=\overline{u(\bar{z})}$. This field glues together left and right components of the Bose field. Let us pass to

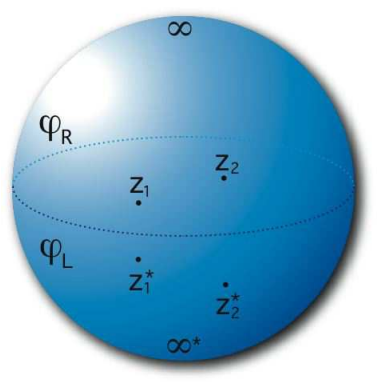

FIG. 1: The double. Left and right sectors are glued together along the branching cut (equator) - a segment of the real axis supporting a particle density. Excitations are symmetrically located in a complex plane.

an imaginary time $t \rightarrow-i t, v \rightarrow i v$, and treat $1 \mathrm{D}$ coordinate $x$ as a real axis of a complex plane $z$. Then right and left currents $\pm \partial_{x} \varphi_{R, L}=\pi \rho \pm i v$ are complex conjugates and may be treated as boundary values of an analytic field in a domain adjacent to the real axis $u(x \pm i 0, t)=v \pm i \pi \rho=-i \partial_{x} \varphi_{R, L}$. Then the hydrodynamic equations (45) are seen as boundary values $(z=x \pm i 0)$ of the quantum Hopf-Burgers equation

$$
\partial_{t} u+u \partial_{z} u=0
$$

This is a nonlinear bosonization of free fermions.

The analytical structure allowing to treat the right current as an analytical continuation of the left one across the boundary (the real axis $x$ ) extends to the Calogero model. There, as we argue below, the boundary values of the field $u$ are

$$
u(x \pm i 0)=(1 / \sqrt{\lambda})(v \pm i \lambda \pi \rho)+\alpha_{0} \partial_{x} \log \rho,
$$

The field $u$ consists of positive and negative parts

$$
u=u^{-}(z)+u^{+}(z) \text {. }
$$

The negative part is a Cauchy transform of the density

$$
u^{-}(z)=\sqrt{\lambda} \int d x \frac{\rho(x)}{x-z} .
$$

It is an analytic function in a plane cut by a segment of a real axis supporting the density. Its boundary values $u^{-}(x \pm i 0)=\pi \sqrt{\lambda}\left(\rho^{H} \pm i \rho\right)$, where $\rho^{H}=$ $(1 / \pi) P \int d x^{\prime} \rho\left(x^{\prime}\right) /\left(x^{\prime}-x\right)$ is a Hilbert transform of the density. A positive part $-u^{+}(z)$ is an analytic continuation of the real function $u^{+}(x)=\left(1 / \sqrt{\lambda}\left(v-\lambda \pi \rho^{H}\right)+\right.$ $\alpha_{0} \partial_{x} \log \rho$ from the real axis. It is analytic only in the vicinity of the real axis, having singularities elsewhere.

Let us mark two points, one inside, another outside of the domain, where both $u^{+}$and $u^{-}$are analytic, and expand the fields around these points. For simplicity let us talk about the rational case. The natural points are $z=0$ and $z=\infty$. We have

$$
u^{-}(z)=-\sum_{n \geq 0} a_{n} z^{-n-1}, u^{+}(z)=-\sum_{n \geq 1} a_{-n} z^{n-1} .
$$

The modes $a_{n}$ are real and Hermitian. Positive modes are moments of density (collective variables) $a_{n}=$ $\sqrt{\lambda} \int x^{n} \rho(x) d x$ and negative modes are canonically conjugated to positive ones $\left[a_{n}, a_{-m}\right]=-n \delta_{n, m}$. The zeromode is the number of particles $a_{0}=\sqrt{\lambda} N$. It does not change in time. The field $u(z)$ is a current of a real canonic Bose field $\varphi=\varphi^{+}+\varphi^{-}$, where $\partial_{z} \varphi^{ \pm}(z)=u^{ \pm}(z)$.

Let us notice a difference between the Bose field (8) and a Bose field used in conventional bosonization of free fermions with a linearized spectrum. In the former, the time $t$ is not a part of a $z$-plane, but describes an evolution of a real holomorphic field $u(z, t)$. In the latter, time and space form a complex coordinate $x+i t$ of the radial quantization. Complex $z$-plane has an analytical structure similar to ones used in boundary CFT and is sometime referred to as (Schottky) double.

\section{Quantum Benjamin-Ono equation is defined as}

$$
\partial_{t} u+\partial_{z}\left(\frac{1}{2} u^{2}+\alpha_{0} \partial_{z}\left(u^{+}-u^{-}\right)\right)=0,
$$

where we rescaled time $t \rightarrow t / \sqrt{\lambda}$. As the Hopf-Burgers equation (6), it is a quantum equation acting in the Fock space spanned by modes $a_{n}$. QBO is integrable. It has infinite number of conserved integrals. The first four are $u$ itself, momentum density $T(z)=u^{2} / 2+i \alpha_{0} \partial\left(u^{+}-u^{-}\right)$, the Hamiltonian

$$
H=\oint \frac{d z}{4 \pi i}\left[\frac{1}{3} u^{3}+\alpha_{0} u \partial_{z}\left(u^{+}-u^{-}\right)\right]
$$

and the fourth integral

$$
I_{4}=\oint \frac{d z}{2 \pi i}\left[\frac{u^{4}}{4}-\frac{3 \alpha_{0}}{2} u^{2} \partial_{z}\left(u^{+}-u^{-}\right)+2 \alpha_{0}^{2}\left(\partial_{z} u\right)^{2}\right]
$$

Here the integration goes around the real axis. Below we show that (910) are equivalent to the CM (11).

The classical BO equation originally appeared in hydrodynamics of stratified fluids. There the real harmonic 
function $u(x, y)$ on a half plane evolves following a timedependent boundary condition on the real axis [7]

$$
\partial_{t} u+u \partial_{x} u+\partial_{x}^{2} u^{H}=0 .
$$

The Hilbert transform $u^{H}=(1 / \pi) P \int d x^{\prime} u\left(x^{\prime}\right) /\left(x^{\prime}-x\right)$ can be seen as $u^{H}=i\left(u^{+}-u^{-}\right)$with respect to the one marked point at infinity. Contrary, our BOequation has two the marked points $z=0$ and $z=$ $\infty$. The term $\left(u^{+}-u^{-}\right)=-i u_{C}^{H}$ in (9) can be seen as a Hilbert transform with respect to the contour $C$ embracing a support of the density (real axis) $u_{C}^{H}(z \in C)=\frac{1}{\pi} P \oint_{C} \frac{u\left(z^{\prime}\right)}{z^{\prime}-z} d z^{\prime}$. This difference is not an obstacle to an almost automatic extension of known solutions of the traditional BO equation. Remarkably, this extension carries over to the quantum case as well.

5. Quantum Hirota equation. Typically, integrable equations can be written in the bilinear (Hirota's) form (see for a review 10, 11]). Apparently, bilinear forms exist also for quantum integrable equations. Let us introduce a vertex operator $\Psi_{\sigma}=\exp (-\sigma \varphi)$, where $\sigma=\sqrt{\lambda}$ or $\sigma=-1 / \sqrt{\lambda}$, and consider its positive and negative parts

$$
\psi^{-}=e^{\sigma \varphi_{-}}, \quad \psi^{+}=e^{-\sigma \varphi_{+}} .
$$

The bilinear quantum Hirota equation

$$
D_{t} \psi^{-} \cdot \psi^{+}=-\frac{1}{2 \sigma} D_{z}^{2} \psi^{-} \cdot \psi^{+},
$$

is an equivalent form of QBO (9). Hirota's derivatives are defined as $D_{x}^{n} a \cdot b=\left.\left(\partial_{x}-\partial_{x^{\prime}}\right)^{n} a(x) b\left(x^{\prime}\right)\right|_{x=x^{\prime}}$. The proof is straightforward.

The Hirota equation (14) is a compact form of OPE (2), extended for Calogero model. The resemblance to the Schrödinger equation further clarifies the origin of QBO. It is especially transparent for free fermions $\lambda=1$. There, the vertex operator $\Psi_{\sigma}$ is a bosonized form of a fermion creation $(\sigma=1)$ and anihilation $(\sigma=-1)$ operators. The Schrödinger equation for free fermions $\partial_{t} \Psi=$ $\frac{1}{2} \partial_{z}^{2} \Psi$ transforms to Hirota equation (14) by an elementary algebra. A known duality between particles and holes $a_{n} \rightarrow a_{-n}$ under a transformation $\sqrt{\lambda} \rightarrow-1 / \sqrt{\lambda}$ is especially transparent in the bilinear form.

6. Quantum hydrodynamics and QBO-equation. Relation to hydrodynamics is obtained by specializing BOequation on the real axis. Computing the jump (discontinuity) of the l.h.s. of (9) across the real axis we obtain the continuity equation (4) with the current $j=\rho v=$ $(T(x+i 0)-T(x-i 0)) /(2 \pi i \sqrt{\lambda})$. It gives the announced connection (7) between the field $u$ and velocity. The sum of $\mathrm{BO}$ equations taken on two sides of the real axis gives the Euler equation (5) with the energy density

$$
\epsilon=\frac{1}{6}(\pi \lambda \rho)^{2}+\frac{\alpha_{0}^{2}}{2}\left(\partial_{x} \ln \rho\right)^{2}+\pi \alpha_{0} \lambda \partial_{x} \rho_{H} .
$$

Eqs. (4515) have been derived in Ref. 5] within the collective field theory approach.
7. Pole dynamics of quantum Benjamin-Ono equation and Calogero model. Let us now show that QBO is equivalent to the Calogero model.

An important class of solutions of classical integrable nonlinear equations is given by a moving pole ansatz. These solutions have a form $u=\sum_{i=1}^{N} f\left(z-x_{i}(t)\right)$, where $f(x)$ is an elliptic function, or its trigonometric or rational degeneration (see, e.g., [7]). Then nonlinear equation is reduced to a certain, also integrable, many body problem of $N$ particles with coordinates $x_{i}$. In most cases it is the Calogero model and its decendents. In Ref. [8] similar fact has been noticed for the classical BOequation (12). The complex poles $x_{i}(t)$ of a pole ansatz $u(x, t)=-\sum_{i}\left(\frac{i \lambda}{x-x_{i}}-\frac{i \lambda}{x-\bar{x}_{i}}\right)$ move according to a complexified classical Calogero equation

$$
\ddot{x}_{i}=2 \sum_{j, j \neq i} \frac{\lambda^{2}}{\left(x_{i}-x_{j}\right)^{3}}
$$

under the condition that $\operatorname{Im} x_{i}>0$ at an initial time.

Our version (21) of the classical BO equation produces a genuine Calogero model where coordinates of particles (poles) are real. Remarkably, the pole ansatz carries over to the quantum case. Pole ansatz is perhaps a simplest way to see the equivalence between CM and QBO.

The algebra is straightforward and can be carried out for both QBO and quantum Hirota equation. We briefly describe the main steps starting from the QBO (9).

We look for a solution in the form

$$
u^{-}(z, t)=-\sqrt{\lambda} \sum_{i=1}^{N} \frac{1}{z-x_{i}(t)}, \quad x_{i}=\text { Real }
$$

and show that the dynamics of the poles $x_{i}$ obey quantum Calogero model with $N$ particles. The residue in (17) is controlled by the asymptote at infinity $u^{-}(z) \sim$ $(-\sqrt{\lambda} N) / z$. At the pole ansatz the vertex operator (13) at $\sigma=-1 / \sqrt{\lambda}$ has simple zeros at positions of particles $\psi^{-}=\prod_{j}\left(z-x_{j}\right)$.

We plug (17) into the negative part of (9) and assume $\left[u^{+}\left(x_{i}\right), x_{j}\right]=-\delta_{i j} / \sqrt{\lambda}$, which yield canonical commutation relations for $u^{ \pm}$as $N \rightarrow \infty$. Using formulas $\left(u^{-} u^{+}\right)^{+}=-\sum_{j} \frac{\sqrt{\lambda}}{z-x_{j}}\left(u^{+}(z)-u^{+}\left(x_{j}\right)\right)$ and $\left(u^{-} u^{+}\right)^{-}=$ $-\sum_{j} \frac{\sqrt{\lambda}}{z-x_{j}} u^{+}\left(x_{j}\right)$, we obtain that a rational function

$$
\begin{aligned}
& \frac{1}{z-x_{j}} \dot{x}_{j} \frac{1}{z-x_{j}}+\sqrt{\lambda} \sum_{k, k \neq j} \frac{1}{\left(x_{j}-x_{k}\right)\left(z-x_{j}\right)^{2}} \\
& +\frac{1}{\sqrt{\lambda}} \frac{1}{\left(z-x_{j}\right)^{3}}-\frac{1}{\left(z-x_{j}\right)^{2}} u^{+}\left(x_{j}\right)=0
\end{aligned}
$$

vanishes. Both second and third order poles vanish if $\dot{x}_{j}=p_{j}$ with $\left[p_{i}, x_{j}\right]=-\delta_{i j} / \sqrt{\lambda}$ and

$$
p_{j}=u^{+}\left(x_{j}\right)-\sqrt{\lambda} \sum_{k, k \neq j} \frac{1}{x_{j}-x_{k}} .
$$


Eq. (19) is a finite $N$ version of the equation (7). Differentiating (19) over time and using the positive part of (9), we obtain after some work Eq. (16) with $\lambda^{2} \rightarrow \lambda(\lambda-1)$ (where time is scaled back to the original real time).

Few comments are in order: (i) The pole ansatz (17) represents positive modes $a_{n}=\sqrt{\lambda} \sum_{j} x_{j}^{n}$. Negative modes are represented in terms of momenta and coordinates of particles through (19). Canonical commutation relations between positive and negative modes appear only in the field theory limit. For a finite number of particles they are replaced by the conditions $\left[u^{+}\left(x_{i}\right), x_{j}\right]=$ $-\delta_{i j} / \sqrt{\lambda}$ used in the cancelation of poles; (ii) Similar manipulations in the classical case produce Calogero model with interaction $\lambda^{2}\left(x_{i}-x_{j}\right)^{-2}$. A quantum shift $\lambda^{2} \rightarrow \lambda(\lambda-1)$ comes from pulling momentum operator $p_{j}$ to the right in the first term of (18); (iii) A trigonometric ansatz $u^{-}(z, t)=-\sqrt{\lambda} k \sum_{i} \cot \left(k\left(z-x_{i}(t)\right)\right.$ leads to the Calogero-Sutherland (trigonometric) model.

8. Solitons and integrability Let us now consider the large $N$ limit and assume that there is a non-zero background density of particles. Then, alternatively, one looks for the pole ansatz for $u^{+}$with a finite number of poles symmetrically located with respect to the real axis (see Fig 1)

$$
u^{+}(z, t)=\sqrt{\lambda} \sum_{i=1}^{n}\left(\frac{1}{z-z_{i}}+\frac{1}{z-z_{i}^{*}}\right) .
$$

In this case the vertex operator (13) at $\sigma=-1 / \sqrt{\lambda}$ is $\psi^{+}(z)=\prod_{i}\left(z-z_{i}\right)$ and has zeros at positions of poles. 2] These poles also move according to quantum Calogero model. They correspond to excitations of a system with a continuous density. Accordingly $u^{-}(z)$ has a branch cut on the real axis. Solutions (states) with well-separated poles $z_{i}$ of $u_{+}(z, t)$ are quantum analogs of solitons known in classical nonlinear equations. A semiclassical onesoliton solution of the Euler equation (5) studied in Ref. [4, 12, 13], corresponds to a single pole in (20) and can be found from (7).

Existence of solitons signals that the QBO is integrable. Indeed, the known Lax representation for the classical BO equation (12) extends to the quantum BO equation on the double (9) with minor changes. In particular, the pole ansatz converts Lax operators of the quantum BO equation to known Lax operators of quantum Calogero model. The same is true for the conserved integrals, Bäklund transformation, etc. In a standard way these tools provide multi-soliton, periodic, and shockwave solutions for quantum hydrodynamics.

10. Semiclassical limit of $B O$ equation and nonlinear transport in quantum systems. Classical BO equation may be useful in studying nonlinear effects in transport in low-dimensional quantum systems. It describes a semiclassical evolution of long wave packets of density. The semiclassical limit is subtle and requires certain care. In nonlinear quantum equation (9) a simple replacement of a quantum field $u$, by classical fields is incorrect. The correct result is obtained by shifting $\alpha_{0} \rightarrow \sqrt{\lambda} / 2$ in (9)

$$
\partial_{t} u+\partial_{z}\left(\frac{1}{2} u^{2}+(\sqrt{\lambda} / 2) \partial_{z}\left(u^{+}-u^{-}\right)\right)=0
$$

In particular, the dispersive (last) term in (21) does not vanish for free fermions at $\lambda=1$, while it vanishes in the quantum version (6). The equation (21) (or rather a corresponding classical action) must be understood as a leading term of the gradient expansion of the effective action, with the first order correction due to zero fluctuations. It can be summurized as $\left\langle u^{2}\right\rangle-\langle u\rangle^{2}=$ $\sqrt{\lambda} \partial_{z}\left\langle u^{+}-u^{-}\right\rangle$, where we average over the coherent state with a given density. The correction ammounts to the shift $\lambda(\lambda-1) \rightarrow \lambda^{2}$. One can also obtain this quantum correction by comparing the classical (16) and quantum (1) $\mathrm{CM}$ obtained as pole ansatzes of classical and quantum BO equations. The classical pole asatz (17) leads to the classical Calogero equation (16) only when it is plugged into (21) rather than into a naive classical version of (9). The classical dynamics of long wave packets depends on $\lambda$ only through scales. It is described by the classical $\mathrm{BO}$ on the double, and is the same for free fermions and "anyons".

The appearance of the dispersion term (the last term in (21) ) for free fermions has been pointed out by A. Jevicki [4]. It has far reaching consequences. As it is well known, a dispersionless limit of non-linear equations is singular 14]. Almost any smooth initial configuration $u(x, t)$ evolving according to a classical Hopf-Burgers equation (6) (a dispersionless limit of (21) ) develops an unphysical shock wave in finite time. The dispersion being initially small becomes a crucial factor and can not be neglected. A discussion of the role of dispersion in electronic transport, and a number of other interesting topics, like quantum integrability, relation to perturbed conformal field theory etc., is outside of the scope of this letter. We plan to address some of these issues elsewhere.

We have benefited from discussions with O. Agam, E. Bettelheim, A. Mirlin, L. Takhtajan, A. Tsvelik, and A. Zabrodin. Our special thanks to I. Gruzberg, D. Gutman and R. Teodorescu for help, and contributions. P.W. was supported by the NSF MRSEC Program under DMR0213745 and NSF DMR-0220198. P.W. acknowledges support by the Humboldt foundation. The work of AGA was supported by the NSF under the grant DMR-0348358 and by the Theory Institute at BNL.

[1] M. A. Olshanetsky and A. M. Perelomov, Phys. Rep. 94, 6 (1983);

A. P. Polychronakos, Les Houches Lectures, 1998, hep-th/9902157 
[2] H. Awata, Y. Matsuo, S. Odake, J. Shiraishi, Phys. Lett. B 347, 49-55, (1995); Nucl. Phys. B 449, 347-374 (1995).

[3] A. Jevicki and B. Sakita, Nucl. Phys. B165, 511-527 (1980);

B. Sakita, Quantum Theory of Many-variable Systems and Fields. World Scientific, 1985;

[4] A. Jevicki, Nucl. Phys. B376, 75-98 (1992).

[5] I. Andrić, A. Jevicki, and H. Levine, Nucl. Phys. B215, 307 (1983);

I. Andrić and V. Bardek, J. Phys. A 21, 2847 (1988).

6] L. D. Landau, Sov. Phys. ZhETF, 11, 592 (1941).

[7] M. A. Ablowitz and P. A. Clarkson, Solitons, Nonlinear Evolution Equations and Inverse Scattering, London Mathematical Society Lecture Note Series (No. 149), 1991.
[8] H. H. Chen, Y. C. Lee, and N. R. Pereira, J. Phys. Fluids 22, 187 (1979).

[9] M. Stone, editor, Bosonization, World Scientific, Singapore, 1994.

[10] Y. Matsuno, Bilinear Transformation Method, in v. 174, Mathematics in science and engineering, Academic Press, 1984.

[11] A. Zabrodin, Theor. Math. Phys. 113, 1347-1392 (1997).

[12] A. P. Polychronakos, Phys. Rev. Lett. 74, 5153 (1995).

[13] I. Andrić, V. Bardek, L. Jonke, Phys. Lett. B 357, 374 (1995).

[14] Singular Limits of Dispersive Waves, eds. N.M. Ercolani et.al., Plenum Press, New York (1994). 